\title{
Aymon de Montfalcon, ambassadeur de la cour de Savoie et du roi de France : un spécialiste de la négociation
}

\section{Eva Pibiri}

\section{CpenEdition}

Journals

Édition électronique

URL : http://journals.openedition.org/edl/1346

DOI : $10.4000 /$ edl. 1346

ISSN : 2296-5084

Éditeur

Université de Lausanne

\section{Édition imprimée}

Date de publication : 1 décembre 2018

Pagination : 85-106

ISBN : 978-2-940331-69-7

ISSN : 0014-2026

\section{Référence électronique}

Eva Pibiri, «Aymon de Montfalcon, ambassadeur de la cour de Savoie et du roi de France : un spécialiste de la négociation », Études de lettres [En ligne], 3-4 | 2018, mis en ligne le 15 décembre 2020, consulté le 16 décembre 2020. URL : http://journals.openedition.org/edl/1346 ; DOI : https:// doi.org/10.4000/edl.1346 


\section{AYMON DE MONTFALCON, AMBASSADEUR DE LA COUR DE SAVOIE ET DU ROI DE FRANCE: UN SPÉCIALISTE DE LA NÉGOCIATION}

Aymon de Montfalcon servit la Maison de Savoie lors de nombreuses ambassades entre 1471 et 1509 environ. Il fut envoyé de manière récurrente auprès des évêques de Sion au sujet des contestations pour les lieux et châteaux du Bas-Valais occupés par les Valaisans et revendiqués par la Savoie, ainsi que vers les Confédérés pour le renouvellement des ligues. Son rapport privilégié avec les Suisses fut également exploité par le roi Louis XII, afin de les engager à accepter l'alliance avec la France. L'évêque de Lausanne peut ainsi être considéré comme un véritable spécialiste des affaires diplomatiques, bénéficiant d'un double ancrage qui fut utilisé avec profit par les ducs savoyards.

L'activité diplomatique d'Aymon de Montfalcon a déjà été quelque peu abordée par Édouard Rott en 1900 dans son Histoire de la représentation diplomatique de la France auprès des Cantons Suisses ${ }^{1}$, ainsi que par Maxime Reymond en 1920 dans l'article qu'il a dédié à l'évêque de Lausanne dans la Revue d'histoire ecclésiastique suisse ${ }^{2}$; toutefois, c'est par une approche de type biographique et événementiel que le sujet a été traité.

Je me propose ainsi de replacer les ambassades d'Aymon de Montfalcon dans le contexte des pratiques diplomatiques de la fin du Moyen Âge, en m'arrêtant particulièrement sur le type d'affaires traitées, la configuration des ambassades auxquelles il prit part, les raisons de la

I. É. Rott, Histoire de la représentation diplomatique de la France auprès des Cantons Suisses, de leurs alliés et de leurs confédérés, I, 1430-1559.

2. M. Reymond, "Aymon de Montfalcon, évêque de Lausanne, 1491-1517». 
longévité de sa carrière et la documentation qui nous est parvenue pour répondre à ces questions.

\section{La documentation}

Les ambassades d'Aymon peuvent être étudiées au travers de documents de typologies variées conservés notamment aux Archives cantonales vaudoises et aux Archives d'État de Turin. Cette documentation permet d'envisager certaines missions dans leur intégralité, depuis la nomination de l'émissaire jusqu'à la conclusion des pourparlers. Il s'agit ainsi de lettres des princes adressées à Aymon, de lettres de créance et de pouvoirs, d'instructions, de rapports de mission ${ }^{3}$, de traités, d'un contrat de mariage et de lettres de nomination ${ }^{4}$.

La trésorerie générale de Savoie contient également les frais de certaines légations de l'évêque de Lausanne, mais il faut avouer que les informations obtenues par les comptes sont relativement pauvres, car il s'agit dans la grande majorité des cas de mentionner le nom des ambassadeurs, le lieu de destination, parfois le pourquoi de la mission (mais de manière succincte), la durée de l'ambassade et les sommes engagées. Il s'agit, de fait, de résumés des pièces comptables remises au trésorier par les ambassadeurs pour justifier leurs frais et se faire rembourser leurs débours.

Ces textes comptables, fortement réduits, se démarquent de ceux que l'on trouve dans les registres du début et du milieu du $\mathrm{XV}^{\mathrm{e}}$ siècle dans lesquels les frais détaillés des émissaires étaient copiés, devenant ainsi extrêmement précieux pour mieux saisir le déroulement des affaires ${ }^{5}$.

Parmi cette documentation, il faut relever en particulier un registre composite intrigant, conservé aux Archives cantonales vaudoises sous la cote $\mathrm{Ab} 14^{6}$ et intitulé Affaires diplomatiques négociées par Aymon

3. Un fragment du journal d'une mission à Fribourg d'Aymon, entre le 5 et le 20 décembre 1494, rédigé par un membre de son entourage, est publié dans P. Rück, «Un récit de la captivité du Chapitre de Lausanne en février 1537 », p. 60 sq.

4. Les recès de la Diète fédérale donnent aussi des informations sur les ambassades d'Aymon; cf. A. Ph. Segesser (Bearb.), Die Eidgenössischen Abschiede aus dem Zeitraum von 1500 bis 1520 .

5. E. Pibiri, En voyage pour Monseigneur, p. 36-38.

6. Numéroté par M. Reymond de 1 à 133. 
de Montfalcon, qui regroupe des papiers concernant les ambassades d'Aymon entre 1506 et 1509 , en premier lieu pour des affaires auprès des Suisses et de l'évêque de Sion.

Ces documents n'ont pas été réunis au XVI e siècle, tant s'en faut. En effet, ce registre a été composé par Maxime Reymond, directeur à titre intérimaire des Archives cantonales vaudoises (ACV) entre 1915 et 1942, qui réunit des documents individuels, parchemins et papiers, qu'il décrit en 1915 comme des correspondances diverses de l'époque savoyarde et épiscopale se trouvant à l'origine dans un carton. Ce regroupement documentaire eut lieu dans le cadre du travail d'inventaire lié au Plan général de classement des ACV adopté en 1915. Aucun document ne porte de cote ancienne, ce qui tend à démontrer que leur sélection fut opérée sur des masses jusqu'alors non considérées 7 . Si Maxime Reymond fut à l'origine de ce registre composite, il n'en demeure pas moins que ces documents furent conservés, vraisemblablement par Aymon de Montfalcon. En effet, le nombre important de lettres du duc Charles II de Savoie lui étant personnellement adressées tend à nous pousser vers cette hypothèse.

Il s'agissait donc pour l'évêque de Lausanne de garder la trace des missions qu'il avait effectuées et d'en faciliter le suivi. Ainsi, la correspondance avec le duc, les instructions et les rapports de mission côtoient certains doubles des lettres d'Aymon et des autres émissaires envoyées à Charles $\mathrm{II}^{8}$, tout comme des copies de missives de souverains impliqués dans les affaires traitées ${ }^{9}$, un procédé courant entre ambassades de princes alliés ${ }^{10}$.

Cette pratique diplomatique, consistant en la conservation et le classement de la documentation relative aux ambassades, fut utilisée de manière très variable par les cours occidentales qui se contentèrent le plus souvent d'archiver soigneusement les trêves, les traités ou encore les

7. Je tiens à remercier chaleureusement Monsieur Gilbert Coutaz, directeur des $\mathrm{ACV}$, pour m’avoir communiqué ces informations. Voir également G. Coutaz, «Le plan général de classement des Archives cantonales vaudoises de 1915 ", p. 68-70.

8. ACV, Ab 14, fol. 30r, 29 avril 1506.

9. Par exemple, la copie de la lettre du roi de France Louis XII adressée aux avoyers et aux Conseils de Berne, Fribourg et Soleure pour leur demander de servir de médiateurs entre la Savoie et l'évêque de Sion, ACV, Ab 14, fol. 18r, 13 avril 1506.

Io. E. Pibiri, En voyage pour Monseigneur, p. 90. 
contrats, sans y ajouter tous les documents qui avaient contribué à leur réalisation, car ils étaient jugés inutiles ${ }^{11}$.

En ce qui concerne la Savoie, ce type de dossiers diplomatiques, organisés autour d'une affaire en particulier ou des relations avec un prince, existaient déjà dans la première moitié du $\mathrm{XV}^{\mathrm{e}}$ siècle ${ }^{12}$. Ainsi, deux registres débutés en $1426^{13}$ et en $1431^{14}$, s'étendant respectivement jusqu'en 1437 et 1436, concernent les affaires italiennes et notamment les négociations et les traités de paix liés à la ligue conclue par Amédée VIII avec Venise et Florence contre le duc de Milan ${ }^{15}$.

Ces recueils permettaient de vérifier le travail accompli par les émissaires, de faciliter la consultation et l'utilisation des textes, afin de garantir le succès des négociations présentes et futures par le recours aux anciens rapports de mission ou encore à la correspondance.

Cette pratique n'était évidemment pas une spécificité savoyarde, car de tels registres existaient en Angleterre, dès la seconde moitié du XIII ${ }^{\mathrm{e}}$ siècle, tout comme à Florence, à partir de $1431^{16}$. Par contre, à la même époque, la France et la Bourgogne ne possédaient pas de service spécifique pour sauvegarder et archiver la documentation relative aux ambassades. Si certains dossiers diplomatiques furent constitués dans ces deux cours, il s'agit toujours d'une initiative personnelle des ambassadeurs qui conservaient ainsi la trace des négociations auxquelles ils avaient participé ${ }^{17}$, comme le firent vraisemblablement Aymon de Montfalcon et sa chancellerie.

II. En 1880, Pietro Vayra, archiviste aux archives de Turin, mentionnait que seuls les actes publics étaient conservés autrefois par la Maison de Savoie et que toutes les correspondances étaient, après quelques années, détruites car jugées inutiles (cf. É. Charavay, "Rapport sur les lettres de Louis XI et sur les documents concernant ce prince conservés dans les archives de l'Italie, adressé à M. le Ministre de l'instruction publique", p. 461).

I2. E. Pibiri, En voyage pour Monseigneur, p. 169-171.

13. Archivio di Stato di Torino, Corte (AST, C), Paesi, Milanese, Città e ducato, mazzo $2, n^{\circ} 6,1,1426-1437$.

I4. AST, C, Materie politiche per rapporto all'interno, Provvedimenti sovrani, Estratti di Savoia, n $8,1431-1436$.

I5. E. Pibiri, En voyage pour Monseigneur, p. 12-14.

I6. F. Senatore, "Uno mundo de carta», p. 108-110 et 118; D. E. Queller, Early Venetian Legislation on Ambassadors, p. 86.

17. A.-B. Spitzbarth, Ambassades et ambassadeurs de Philippe le Bon, troisième duc Valois de Bourgogne (1419-1467), p. 116 sq.; F. Autrand, Ph. Contamine, «Naissance de la France», p. 110; F. Autrand, "L'enfance de l'art diplomatique», p. 211-213. 


\section{Une longue période d'activité}

Aymon servit les ducs de Savoie bien avant d'être nommé au siège épiscopal en 1491. En effet, son activité diplomatique est déjà attestée au début des années 1470, comme l'indique le texte de sa nomination au conseil ducal en 1471, dans lequel Aymon est qualifié par Amédée IX de «très cher orateur [...] qui a œuvré depuis longtemps pour de durs négoces secrets ${ }^{18}$. Il assuma des missions jusqu'en 1509 au moins ${ }^{19}$, alors qu'il était âgé de 66 ans environ, soit huit ans avant son décès ${ }^{20}$. L'état de santé d'Aymon, qui est évoqué dans les lettres de Charles II dès $1507^{21}$, fut sans doute l'une des raisons de la suspension de ses activités.

Il fut ainsi un émissaire de la cour de Savoie pendant près de 40 ans, ce qui l'amena à servir plusieurs ducs, régentes et membres de la famille ducale, dans un contexte où les principats savoyards furent parfois très brefs de par le décès précoce de certains ducs, laissant des héritiers mineurs sous l'autorité de leur mère régente ${ }^{22}$. Aymon fut ainsi choisi comme émissaire par Amédée IX, son frère Louis de Savoie, roi de Chypre, Charles I ${ }^{\text {er }}$, François de Savoie, évêque de Genève, Blanche de Montferrat, duchesse de Savoie, Philippe II, Philibert le Beau, Marguerite d'Autriche et Charles II.

Les missions d'ambassade d'Aymon de Montfalcon étaient des missions temporaires, car, à l'époque qui nous intéresse, les ambassades dites permanentes n'étaient de loin pas encore la norme. Si des légats résidents furent dépêchés, au milieu du XV $\mathrm{XV}^{\mathrm{e}}$ siècle, par Venise, Florence et Milan ${ }^{23}$ dans les cours les plus prestigieuses, notamment celles du pape à Rome et du roi de France, les légations ponctuelles restèrent un des piliers de la diplomatie des États.

I8. ACV, CII 206, 11 décembre 1471. Texte traduit par E. Pibiri.

19. La dernière ambassade d'Aymon de Montfalcon date, à ma connaissance, d'octobre 1509. Aymon fut envoyé à Berne pour traiter, notamment, de l'affaire Dufour, cf. ACV, Ab 14, fol. 130r; A. Ph. Segesser (Bearb.), Die Eidgenössischen Abschiede aus dem Zeitraum von 1500 bis 1520, p. 472 sq. Dès 1513, Sébastien de Montfalcon, neveu d'Aymon, fut d'ailleurs nommé coadjuteur de son oncle par Léon X (cf. "Sébastien de Montfalcon", in Helvetia sacra, I/4, p. 148 sq. Sur l'affaire Dufour, cf. infra, n. 50.

20. "Aymon de Montfalcon", in Helvetia sacra, I/4, p. 146-149.

2I. ACV, Ab 14, fol. 46r; M. Reymond, "Aymon de Montfalcon, évêque de Lausanne, 1491-1517", p. 105.

22. Th. Brero, Rituels dynastiques et mises en scène du pouvoir, p. 9-29.

23. F. Senatore, "Uno mundo de carta", p. 32 et 43 sq. 
Chaque envoi d'ambassade était le résultat d'une décision du prince et de son Conseil. La composition de la légation faisait l'objet d'un choix réfléchi, les personnes les plus susceptibles de mener à bien la mission étaient ainsi sélectionnées ${ }^{24}$.

Aymon ne se limita pas à défendre les intérêts de la Maison de Savoie. Il effectua également des missions diplomatiques pour le roi de France, Louis XII, entre 1505 et $1509^{25}$. Ses liens avec la couronne étaient anciens, car Aymon avait déjà été conseiller du roi Charles VIII en $1489^{26}$. Servir deux princes n'était pas une pratique diplomatique inhabituelle, au contraire, un ancrage dans une autre cour pouvait devenir un argument de sélection au moment d'envoyer une ambassade $^{27}$.

\section{Un ambassadeur de prestige et spécialisé}

Les ambassades d'Aymon peuvent être classées en deux grandes catégories. En premier lieu, des missions de prestige qui consistaient à représenter le prince lors de la conclusion de mariages par exemple, de traités de paix ou encore de la prestation de l'hommage à l'empereur.

L'évêque de Lausanne fut ainsi choisi en septembre 1496 par le duc Philippe II pour se rendre auprès de l'empereur Maximilien à Vigevano, alors que la situation était tendue entre les deux princes, la Savoie se trouvant géographiquement et politiquement entre la France et l'Empire qui s'affrontaient en Italie. L'ambassade savoyarde ne comptait pas moins de 150 personnes et montures ${ }^{28}$. La même année, en novembre, lorsque le duc dépêcha son fils Philibert le Beau, alors âgé de 16 ans, auprès du

24. J.-M. Moeglin, «La place des messagers et des ambassadeurs dans la diplomatie princière à la fin du Moyen Âge», p. 12 et 14; J.-M. Moeglin (dir.), S. Péquignot (coll.), Diplomatie et "relations internationales» au Moyen Âge (IXe-XVe siècle), p. 374-380 et 389-418.

25. É. Rott, Histoire de la représentation diplomatique de la France auprès des Cantons Suisses, de leurs alliés et de leurs confédérés, I, p. 107, 152-154 et 166-168.

26. ACV, CI c3, 12 juin 1489.

27. E. Pibiri, «Le personnel diplomatique d'Amédée VIII de Savoie entre France et Bourgogne au temps de la guerre de Cent Ans (1410-1440)».

28. Archivio di Stato di Torino, Sezioni riunite, camerale Savoia, inventario 16, Trésorie générale de Savoie (AST, TGS), $\mathrm{n}^{\circ}$ 150, 1496-1497, fol. 273r-274r; F. Gabotto, Lo Stato sabaudo da Amedeo VIII ad Emanuele Filiberto, t. III, p. 25 sq. 
roi de France Charles VIII qui se trouvait à Lyon, Aymon fit également partie de cette délégation visant à maintenir le fragile équilibre dans lequel se trouvait la Savoie ${ }^{29}$.

De même, en août 1501, ce fut à l'occasion du mariage de Philibert le Beau et de Marguerite d'Autriche qu'Aymon se trouva à la tête de l'imposante délégation, pas moins de 250 montures selon le chroniqueur bourguignon Jean Molinet, chargée de se rendre à Bruxelles pour ramener la future mariée en Savoie ${ }^{30}$. S'il est difficile de confirmer les chiffres avancés par l'historiographe, car la trésorerie générale de Savoie ne mentionne pas le nombre de personnes composant la légation, il n’en demeure pas moins que la somme remise aux ambassadeurs avant leur départ pour Bruxelles implique une légation nombreuse: 12'320 florins, dont 3000 uniquement pour Aymon ${ }^{31}$. Le mariage par procuration entre Marguerite et le Bâtard René de Savoie ${ }^{32}$, représentant du futur époux, fut d'ailleurs célébré à Salins par Aymon en personne le 28 novembre $1501^{33}$.

Si l'évêque de Lausanne fut certes à la tête de ces délégations, il était également accompagné par d'autres hauts dignitaires dont l'autorité et le prestige cumulés conféraient une solennité particulière à l'ambassade. Il s'agissait ainsi de légations composées d'un nombre élevé de personnes et de montures, nous l'avons vu, afin d'honorer le destinataire de la légation, mais également de manifester la puissance ducale. Par exemple, dans le cadre de l'ambassade devant ramener Marguerite d'Autriche en Savoie, Aymon partit, entre autres, avec le maréchal de Savoie Hugues de La Palud, comte de Varax, le baron Amédée de Viry, Honoré de Bueil, Claude de Balleyson, Pierre de Bonvillars, président du Conseil de Chambéry, Angelino Provana, président patrimonial, François de Menthon, Alexandre, bâtard de Châteauneuf, et le secrétaire

29. AST, TGS, n 150, 1496-1497, fol. 205v-207v; F. Gabotto, Lo Stato sabaudo da Amedeo VIII ad Emanuele Filiberto, III, p. 27.

30. J. Molinet, Chroniques, II, p. 488.

3I. AST, TGS, n 153, 1500-1502, fol. 310r-312r.

32. Il était un fils illégitime du duc Philippe II de Savoie, cf. R. Crotti Pasi, «Filippo II, duca di Savoia".

33. J. Molinet, Chroniques, II, p. 488; M. Bruchet, Marguerite d'Autriche, duchesse de Savoie, p. 35; AST, TGS n 153 , 1500-1502, fol. 397r-398r et 654r-673v. 
Dufour: des membres éminents du conseil ducal, de la noblesse et de la magistrature savoyardes ${ }^{34}$.

Les ambassadeurs étaient d'ailleurs principalement choisis parmi les conseillers, car ils étaient au courant des affaires politiques en cours et côtoyaient régulièrement leur seigneur. Les barons, les prélats et les nobles étaient ainsi nombreux à prendre part aux négociations diplomatiques, tout comme les légistes qui étaient indispensables au sein des légations pour régler les questions d'ordre juridique. Les ambassades composées de conseillers nobles et de juristes conciliaient parfaitement les deux aspects des négociations. En premier lieu, celui du cérémonial et des usages de la cour, que les seigneurs maîtrisaient parfaitement, où l'accent était mis sur le prestige personnel et social. Dans un second temps, celui de la technique, prise en charge par les hommes de loi, dont le savoir était essentiel ${ }^{35}$.

Si les prélats et les grands seigneurs étaient choisis lors de négociations capitales, ils n'intervenaient toutefois qu'à la fin des pourparlers, pour en parfaire les dispositions et les concrétiser solennellement. Toute la préparation des affaires était assurée par de nombreuses ambassades réduites, composées fréquemment d'un conseiller ou d'un écuyer, d'un juriste et parfois d'un secrétaire, comme ce fut le cas lors du mariage austro-savoyard, où Simon de Chantrans, conseiller, et François Richard, secrétaire, furent mandés, en décembre 1500 déjà, pour travailler à l'accord qui ne fut conclu qu'en été $1501^{36}$.

Ces missions de prestiges ne furent toutefois pas les plus caractéristiques de la carrière diplomatique d'Aymon. En effet, ce dernier s'est surtout illustré par ses nombreuses ambassades auprès des Suisses, notamment entre 1491 et $1509^{37}$, tout comme par celles auprès des

34. AST, TGS n ${ }^{\circ}$ 153, 1500-1502, fol. 310r-312r, 699v-700r; J. Molinet, Chroniques, II, p. 488; J. Dumont, Corps universel diplomatique du droit des gens, IV, partie I, p. 15; F. Gabotto, Lo Stato sabaudo da Amedeo VIII ad Emanuele Filiberto, III, p. 183; M. Bruchet, Marguerite d'Autriche, duchesse de Savoie, p. 31.

35. E. Pibiri, En voyage pour Monseigneur, p. 66.

36. F. Gabotto, Lo Stato sabaudo da Amedeo VIII ad Emanuele Filiberto, III, p. 180.

37. ACV, CII 220 bis; ACV, CII 225 et CII 225 annexe; ACV, Ab 14; AST, TGS, n' 151, 1497-1498, fol. 123r-125r; A. Ph. Segesser (Bearb.), Die Eidgenössischen Abschiede aus dem Zeitraum von 1500 bis 1520, p. 155, 365, 472 sq., 298 et 428; F. Gabotto, Lo Stato sabaudo da Amedeo VIII ad Emanuele Filiberto, II, p. 471, III, p. 52 sq. et 84 sq.; M. Reymond, "Aymon de Montfalcon, évêque de Lausanne, 1491-1517", p. $99-103$. 
évêques de Sion, entre 1489 et $1506^{38}$, au sujet des contestations pour les lieux et châteaux du Bas-Valais occupés par l'évêque et les Valaisans et revendiqués par la Savoie. Les Suisses furent d'ailleurs également impliqués dans ce litige, car ils opérèrent en tant que médiateurs, de même que la France. Nous voyons ainsi Aymon traiter avec les différents évêques de Sion, Josse de Silenen (1482-1496), Nicolas Schiner (14961499) et Mathieu Schiner (1499-1522) ${ }^{39}$.

$\mathrm{Si}$, lors de sa première intervention dans ce conflit en $1489^{40}$, Aymon n'était pas encore évêque, c'est fort de sa nouvelle charge qu'il traita régulièrement avec ses homologues valaisans, directement ou lors de journées réunissant les émissaires des différents partis, de même que les médiateurs. Il se rendit ainsi à Aigle, Monthey et Sion, entre 1493 et 1495 notamment ${ }^{41}$, alors que le ton montait entre le duc de Savoie et Josse de Silenen et que le recours aux armes était proche.

En 1494, la duchesse de Savoie Blanche de Montferrat spécifie d'ailleurs dans les instructions à ses ambassadeurs qu'Aymon bénéficiait d'un statut spécial par rapport à eux, car il connaissait parfaitement le dossier et que, pour ce faire, il était habilité à procéder comme mieux lui semblerait avec l'évêque de Sion ${ }^{42}$.

C'est grâce à un rapport de mission d'Aymon à Charles II, rédigé en avril 1506, que l'on saisit le mieux la nature que pouvaient revêtir des négociations entre deux seigneurs prélats. Aymon mentionne en effet avec force détails qu'au cours de son ambassade à Sion, l'évêque Mathieu Schiner et lui entendirent la messe ensemble, burent de la malvoisie en sortant de l'église, firent bonne chère, participèrent à la fête des dames à

38. ACV, CII 216, 21 novembre 1489; ACV, CII 219 bis, 1493-1494; ACV, Ab 14, fol. $25 \mathrm{r}$ et 27r ; A. Ph. Segesser (Bearb.), Die Eidgenössischen Abschiede aus dem Zeitraum von 1500 bis 1520, p. 342 et 344; F. Gabotto, Lo Stato sabaudo da Amedeo VIII ad Emanuele Filiberto, II, p. 471 et 497-499; M. Reymond, "Aymon de Montfalcon, évêque de Lausanne, 1491-1517", p. 104.

39. "Jost von Silenen, 1482-1496"; "Matthäus Schiner 1499-1522", in Helvetia sacra, I/5, p. 223 et 233; A. Büchi, Kardinal Matthäus Schiner als Staatsmann und Kirchenfürst, p. 4 sq., 81 sq. et 90-104.

40. ACV, CII 216, 21 novembre 1489.

4I. ACV, CII 219 bis, 1493-1494; F. Gabotto, Lo Stato sabaudo da Amedeo VIII ad Emanuele Filiberto, II, p. 498 sq.; C. Santschi, "Les annales du notaire montheysan Claude Revilliodi, 1490-1525», p. 46.

42. ACV, CII 219 bis, 1493-1494, doc. 2. 
Sion et que l'évêque le logea gracieusement chez lui ${ }^{43}$. Malgré cet accueil chaleureux, Aymon rapporte que l'évêque de Sion lui coupa violemment la parole à plusieurs reprises, alors qu'il lui exposait les revendications ducales; des revendications que Mathieu Schiner rejeta d'ailleurs d'un revers de main en assurant quaucune terre ne serait rendue. La réponse qu'Aymon fit à Mathieu Schiner expose parfaitement l'avantage de choisir l'évêque de Lausanne, prince spirituel et temporel, tout comme l'était l'évêque de Sion, pour que les négociations se fassent, non seulement sur un pied d'égalité, mais également qu'il soit possible de tenir tête au Valaisan. Ainsi, Aymon lui rétorqua avec aplomb et agacement qu'il entendait toujours la même "chanson" avec lui, qu'il ne fallait pas qu'il abuse, car jamais ce pays ne lui serait laissé et qu'il était impossible qu'il puisse le garder ${ }^{44}$.

La fonction épiscopale d'Aymon de Montfalcon fut également un critère de choix pour traiter avec les Suisses. Son autorité s'étendait en effet sur Berne, Fribourg et Soleure qui dépendaient de son diocèse. Ceux-ci le nomment d'ailleurs parfois dans les textes "notre seigneur l'évêque " ${ }^{45}$. Aymon dirigea ainsi une bonne dizaine d'ambassades auprès des Suisses entre 1491 et $1509^{46}$, en premier lieu auprès des Bernois et des Fribourgeois.

Le renouvellement de l'alliance avec les Confédérés fut l'un des gros dossiers d'Aymon ${ }^{47}$, mais de loin pas le seul qu'il dut gérer. Aymon apaisa aussi, en 1491, le mécontentement des Bernois face à la mainmise de la Savoie sur la nomination de l'évêque de Genève, Antoine Champion ${ }^{48}$. Il traita, de même, de l'arbitrage des Suisses entre la Savoie et les évêques de Sion ${ }^{49}$ et dut négocier âprement avec les Confédérés,

43. ACV, Ab 14, fol. 25r-26v, 27r-v.

44. ACV, Ab 14, fol. 25r.

45. É. Rott, Histoire de la représentation diplomatique de la France auprès des Cantons Suisses, de leurs alliés et de leurs confédérés, I, p. 153.

46. Cf. supra, n. 37.

47. ACV, Ab 14, fol. 30r-32r, 66r-69v, 113r-117r; CII 220 bis; CII 225; CII 225 annexe; AST, TGS, $n^{\circ}$ 151, 1497-1498, fol. 119r-125r; n 150, 1496-1497, fol. 439-441r, 443v, 455r; F. Gabotto, Lo Stato sabaudo da Amedeo VIII ad Emanuele Filiberto, II, p. 471, III, p. 52 sq. et 84 sq.

48. F. Gabotto, Lo Stato sabaudo da Amedeo VIII ad Emanuele Filiberto, II, p. 471.

49. ACV, ACV, Ab 14, fol. 47r-49r; CII 219 bis; F. Gabotto, Lo Stato sabaudo da Amedeo VIII ad Emanuele Filiberto, II, p. 497 sq.; M. Reymond, "Aymon de Montfalcon, évêque de Lausanne, 1491-1517», p. 99 sq. 
en 1508 et 1509 , à la suite des faux documents que le secrétaire ducal Jean Dufour leur avait vendus, des documents qui stipulaient, entre autres, que le duc Charles I ${ }^{\text {er }}$ leur avait légué des sommes importantes et avait consenti en leur faveur une hypothèque sur plusieurs terres de son duché ${ }^{50}$.

Ce rapport privilégié de l'évêque de Lausanne avec les Suisses fut également exploité à trois reprises par le roi de France Louis XII entre 1505 et 1509 , notamment parce qu'il n'y avait pas d'ambassade permanente de France auprès d'eux. Aymon était proche des souverains aux fleurs de lys, car il était déjà conseiller de Charles VIII en 1489. Ainsi, en novembre et décembre 1505, Amon fut mandé à Berne par le roi afin d'engager les conseillers de cette ville à accepter l'alliance avec la France ainsi que des pensions royales, tandis qu'en 1509, l'évêque de Lausanne travailla activement au renouvellement de l'alliance entre le roi et les Confédérés, alors qu'il avait été compromis par des négociations secrètes engagées par le roi Charles VIII. Dans ce contexte extrêmement tendu, les ambassadeurs de France n'osèrent pas dépasser Lausanne sans sauf-conduit et demandèrent à Aymon d'aller en personne présenter les articles du renouvellement d'alliance élaborés par le conseil du roi ${ }^{51}$. Aymon assista ainsi, à Lucerne, à la diète du 16 avril 1509, à celle de Berne le 14 mai, et encore à Lucerne les 13 et 27 juin, avec Gaucher de Dinteville, ambassadeur du roi de France, cette fois-ci, mais sans parvenir à un accord ${ }^{52}$.

Quand il ne servait pas directement les intérêts de la France par des missions, Aymon favorisait les ambassadeurs du roi en les accueillant à Lausanne et en leur servant d'intermédiaire pour communiquer avec les Suisses. Ce fut le cas en mars 1507, lorsque Aymon écrivit à l'avoyer et

50. A. Ph. Segesser (Bearb.), Die Eidgenössischen Abschiede aus dem Zeitraum von 1500 bis 1520, p. 472 sq. ; É. Rott, Histoire de la représentation diplomatique de la France auprès des Cantons Suisses, de leurs alliés et de leurs confédérés, I, p. 175 sq.; T. Scott, The Swiss and their Neighbours, chap. "The Dufour Affair", p. 89-95.

5I. É. Rott, Histoire de la représentation diplomatique de la France auprès des Cantons Suisses, de leurs alliés et de leurs confédérés, I, p. 107 et 152-154.

52. A. Ph. Segesser (Bearb.), Die Eidgenössischen Abschiede aus dem Zeitraum von 1500 bis 1520, p. 458 sq., 464 et 472 sq.; M. Reymond, "Aymon de Montfalcon, évêque de Lausanne, 1491-1517», p. 105, 110, 115 et 165-168. 
au Conseil de Fribourg pour leur demander d'envoyer des messagers à cheval pour conduire les ambassadeurs du roi à Lucerne ${ }^{53}$.

Si le roi de France utilisa Aymon pour ses liens avec les Suisses, le duc de Savoie profita aussi des bonnes relations que l'évêque entretenait avec Louis XII pour favoriser des négociations. En effet, en mai 1507, Charles II dépêcha Aymon vers le roi afin que la Savoie puisse s’approvisionner en blé dans le Languedoc, le Dauphiné et en Provence, obtenir une pension royale pour le duc et enfin solliciter l'arbitrage de Louis XII dans l'affaire qui opposait Charles II à son cousin, le bâtard René, qui réclamait que le comté de Villars en Bresse lui soit restitué, après en avoir été dépossédé en 1502 par Philibert le Beau ${ }^{54}$.

Aymon fut ainsi un maillon essentiel de la diplomatie savoyarde. Par la récurrence avec laquelle il fut envoyé auprès des Suisses et des évêques de Sion, il peut être considéré comme un véritable spécialiste ${ }^{55}$, mâ̂trisant parfaitement ces affaires délicates de longue durée. Un tel suivi facilitait les négociations, tant dans leurs aspects pratiques que diplomatiques, donnait lieu à une meilleure connaissance du dossier et contribuait à instaurer un climat de confiance entre les partis. Ces missions répétées permettaient également de s'appuyer sur un réseau d'officiers connus, susceptibles de favoriser le succès des missions, notamment par une bonne information. Au XV siècle déjà, les princes avaient saisi les bénéfices qu'ils pouvaient tirer de cette pratique et eurent tendance à privilégier la continuité des rapports en envoyant à maintes reprises les mêmes émissaires auprès d'un seigneur et en se servant des doubles ancrages ${ }^{56}$, comme ce fut le cas pour Aymon et ses liens avec la France.

53. É. Rott, Histoire de la représentation diplomatique de la France auprès des Cantons Suisses, de leurs alliés et de leurs confédérés, I, p. 108 et 155; M. Reymond, "Aymon de Montfalcon, évêque de Lausanne, 1491-1517», p. 104.

54. ACV, Ab 14, fol. 40-42, 5 mai 1507. Sur cette affaire voir: Th. Brero, Rituels dynastiques et mises en scène du pouvoir, p. 23 sq. et 27.

55. Sur la question des ambassadeurs spécialistes, voir en dernier lieu J.-M. Moeglin (dir.), S. Péquignot (coll.), Diplomatie et "relations internationales» au Moyen Âge (IX ${ }^{e}$ $X V^{e}$ siècle), p. 411-417.

56. Cf. supra, n. 27. 


\section{Les qualités de l’ambassadeur}

Si les critères de choix des émissaires étaient évalués de manière particulière et individuelle, selon leur fonction, leur réseau ou encore leur double ancrage, leurs capacités propres étaient également fondamentales. Un discours théorique sur les qualités et les vertus requises indispensables à l'ambassadeur fut d'ailleurs formulé, pour la première fois au Moyen Âge, en 1436 par le juriste français Bernard du Rosier, futur archevêque de Toulouse, dans son Ambaxiatorum brevilogus ${ }^{57}$. La loyauté, la discrétion, l'éloquence, l'impassibilité, la patience, l'humilité, la prestance, la capacité à écouter et la confiance devaient notamment faire partie des qualités possédées par l'ambassadeur idéal ${ }^{58}$.

Les lettres de nomination et les missives ducales, notamment, envoyées à Aymon donnent des indications sur la manière dont il était considéré par les princes de Savoie et sur les vertus qui lui étaient reconnues. Ainsi, l'on retrouve la fidélité, la confiance, la probité, la prudence, la sagacité, la prévoyance, mais sont également mises en avant la science, les connaissances et l'expérience d'Aymon, docteur en droit canon, tout comme sa grande élocution ${ }^{59}$. Sur ce point, le rapport d'ambassade de la journée qui eut lieu à Aigle entre les représentants de la duchesse Blanche de Savoie et ceux de l'évêque de Sion, le 12 janvier 1494, au sujet des contestations territoriales savoyardes sur le Bas-Valais, mentionne clairement qu'Aymon prit la parole solennellement au cours de la rencontre ${ }^{60}$.

La confiance dont Aymon bénéficiait à la cour de Savoie, ainsi que le bien-fondé de ses conseils furent également reconnus à maintes reprises. En effet, en 1494, la duchesse Blanche de Montferrat lui écrivit qu'elle se fiait à son jugement et acceptait, comme il l'avait suggéré, de défrayer les ambassadeurs des Ligues afin de favoriser les négociations et que pour ce faire elle avait ordonné au trésorier général de Savoie de prendre en

57. E. Pibiri, En voyage pour Monseigneur, p. 49.

58. A.-B. Spitzbarth, Ambassades et ambassadeurs de Philippe le Bon, troisième duc Valois de Bourgogne (1419-1467), p. 225-236. Cf. également B. Behrens, "Treatises on the Ambassadors written in the Fifteenth and Early Sixteenth Century»; M. Bazzoli, "Ragion di stato e interessi degli stati» et en dernier lieu S. Andretta, S. Péquignot, J.-C. Waquet (éds), De l'ambassadeur.

59. ACV, CII 206, CII 208, CII 210, CII 218, CII 219 bis.

6o. ACV, CII 219 bis, doc. 6. 
charge les dépenses des ambassadeurs, comme l'évêque l'entendait ${ }^{61}$. Il en va de même dans les lettres que Charles II adressa à Aymon. Ainsi, en 1508 et 1509, le duc remercia vivement l'évêque de Lausanne pour les recommandations qu'il lui avait faites dans le cadre des revendications territoriales de René, Bâtard de Savoie. Des recommandations qu'il mentionne d'ailleurs avoir suivies ${ }^{62}$.

C'est toutefois dans une missive de Charles II, datée du 19 juin $1507^{63}$, que les compétences et la valeur d'Aymon de Montfalcon sont le plus manifestes. En effet, dans le cadre d'une énième rencontre avec les Suisses à laquelle le duc souhaitait dépêcher Aymon, Charles II exprime sa satisfaction de pouvoir compter sur l'évêque de Lausanne pour cette mission, alors que ce dernier avait été souffrant. Le duc écrit ainsi à Aymon, d'une manière sans doute quelque peu rhétorique, qu'il est fort joyeux de sa guérison "car il n'est pas homme de son état pour qui il a plus d'affection et pour qui il a le plus de déplaisir quand il va mal» ${ }^{64}$. Il était évidemment surtout satisfait qu'Aymon puisse se rendre auprès des Suisses non seulement, comme il l'indique, "pour la grande confiance qu'il lui témoigne», mais surtout, car il sait qu'Aymon "connaît parfaitement le dossier, qu'il agit toujours bien et mieux que les autres». Pour cette raison, le duc n'avait pas voulu confier cette négociation à un autre émissaire.

\section{Les techniques diplomatiques d'Aymon de Montfalcon}

Les rapports d'ambassade rédigés par Aymon pour rendre compte de l'avancée des négociations décrivent la manière dont il gérait les pourparlers. Avant tout chose, nous voyons l'évêque de Lausanne mener de véritables campagnes de renseignement pour saisir au mieux la situation: il écoute, observe, organise de nombreux repas avec des personnes influentes dont il apprend telle ou telle nouvelle qu'il transmet immédiatement au duc ${ }^{65}$. Par exemple, en novembre 1497, lors de son

\footnotetext{
6I. ACV, CII 219 bis, doc. 4.

62. ACV, Ab 14, fol. 58 r et 110r. Cf. supra, n. 32 et 54 .

63. ACV, Ab 14, fol. 46r.

64. Traduction E. Pibiri.

65. ACV, Ab 14, fol. 30r-31v et 66r-68r.
} 
séjour à Fribourg pour le renouvellement de la ligue entre la Savoie, Berne et Fribourg, l'évêque de Lausanne reçut à son logis pour un repas 72 Fribourgeois en trois jours, pour un montant de 12 florins, tandis qu'à Berne, en janvier 1498, ce ne sont pas moins de 192 repas qui furent offerts à des membres du Conseil pour le prix de 32 florins ${ }^{66}$. Ces sommes furent d'ailleurs facturées au duc de Savoie, la table étant, et est toujours, un haut lieu des affaires diplomatiques.

De même, Aymon n'hésitait pas à offrir des sommes importantes à qui pouvait favoriser sa mission. Ainsi, en décembre 1507, alors qu'il se trouvait à Fribourg, à nouveau pour le renouvellement des alliances ${ }^{67}$, il promit 100 écus aux membres du Conseil de Fribourg "pource qu'ils ont toujiours plus de querelle que les aultres et que nous desirons les gaignier" ${ }^{6}$.

Il insista d'ailleurs auprès de Charles II pour qu'il s'attache, moyennant finance, l'avoyer de Fribourg, François Arsent ${ }^{69}$, généralement contraire à la Savoie:

Je l'ay reduyt de sorte qu'il s'est offert d'estre votre bon et humble serviteur, et espere que tel il sera si a vous ne tient et pourveu qu'il vous plaise le bien traicter, car c'est ung homme de grant credit, de grant sens et bien soubtil, et qui est pour vous faire cy après de grans services ${ }^{70}$.

L'évêque de Lausanne utilisa également sa charge ecclésiastique au profit de la réussite des négociations. Ce fut le cas en novembre-décembre 1505, époque où, alors qu'il se trouvait à Berne pour engager des conseillers de la ville à accepter des pensions du roi de France, Aymon n'hésita pas à délier de leur serment certains d'entre eux qui avaient pourtant juré

66. AST, TGS, n 151, 1497-1498, fol. 119r, 123r.

67. ACV, Ab 14, fol. 66r-67r et 68r-69v.

68. ACV, Ab 14, fol. 69v; M. Reymond, "Aymon de Montfalcon, évêque de Lausanne, 1491-1517», p. 102.

69. E. Tremp, «Arsent, François». Avoyer de 1507 à 1509, il épousa Margareta von Diesbach, fille de Wilhelm von Diesbach. Il fut, à Fribourg, le chef des partisans de la France dont il touchait d'ailleurs une pension.

70. ACV, Ab 14, fol. 69v; M. Reymond, "Aymon de Montfalcon, évêque de Lausanne, 1491-1517», p. 102. 
solennellement de ne plus recevoir de pensions de l'étranger ${ }^{71}$. La fin justifie les moyens!

\section{Un ambassadeur récompensé}

Les mérites diplomatiques d'Aymon, ainsi que ses services pour la Maison de Savoie, furent récompensés en diverses occasions. En effet, en 1471, Amédée IX le nomma conseiller pour avoir été orateur et pour ses immenses services et "choses secrètes ${ }^{72}$. Deux ans plus tard, devenu également conseiller de Louis de Savoie, roi de Chypre, il reçut la charge d'orateur et de procureur à la curie romaine avec un salaire annuel de 500 florins pour tenter de convaincre le souverain pontife d'aider le roi exilé de Chypre à reprendre son royaume perdu ${ }^{73}$.

Les reconnaissances suivirent en 1490 avec la nomination d'Aymon au titre de gouverneur et maître de la chapelle ducale, pour une rétribution de 300 florins par an, pour ses "très grands services». Un poste qui fut confirmé six ans plus tard ${ }^{74}$. Enfin, en 1508, Charles II lui fit remettre 1000 écus d'or en remerciement de ses peines auprès des Cantons suisses et du roi de France, alors qu'Aymon ouvrait sans trêve à ces affaires depuis $1506^{75}$.

\section{Conclusion}

Ambassadeur de prestige lors de grandes légations de représentation, mais également chargé de missions plus épineuses, Aymon de Montfalcon fut un homme rompu aux affaires diplomatiques, qui en connaissait tous les rouages.

7I. É. Rott, Histoire de la représentation diplomatique de la France auprès des Cantons Suisses, de leurs alliés et de leurs confédérés, I, p. 152 sq.

72. ACV, CII 206, 11 décembre 1471.

73. ACV, CII 208, 24 décembre 1473. Sur Louis de Savoie et Chypre voir E. Pibiri, «Histoire de femme, histoire d'État», p. 449-451.

74. ACV, CII 218, $1^{\text {er }}$ juin 1490 et 15 novembre 1496.

75. AST, C, Materie politiche per rapporto all'interno, Protocolli dei Notai della Corona, Protocolli Ducali Serie Rossa, n 135, fol. 90. 
Suivant des dossiers sur la longue durée, tant celui des rapports helvetico-savoyards que celui des liens avec les évêques de Sion, l'évêque de Lausanne apparaît comme un véritable spécialiste dont les capacités furent reconnues et valorisées par les ducs de Savoie.

Docteur en droit, seigneur temporel et spirituel, conseiller, Aymon conciliait toutes les facettes nécessaires aux négociations, tant par une maîtrise des usages de cour et du cérémonial que des pratiques juridiques. $\mathrm{Si}$, comme tous les ambassadeurs, Aymon reçut des instructions précises pour ses missions, il bénéficia toutefois d'une grande marge de manœuvre de par la confiance dont il jouissait auprès des ducs savoyards. Prélat choisi par ces derniers pour traiter d'égal à égal avec les princes, il n'en demeure pas moins que même en défendant leurs intérêts, l'évêque de Lausanne demeurait un grand seigneur qui tolérait mal qu'on l'interrompe ou qu'on lui manque de respect, comme nous l'avons vu avec la réponse peu diplomatique qu'il asséna à l'évêque de Sion Mathieu Schiner en 1506, une réponse qui aurait pu mettre le feu aux poudres, mais qui expose également la délicate tension entre le service au prince et le prestige personnel ${ }^{76}$.

C'est peut-être dans ce contexte qu'il faut replacer les instructions adressées à Aymon et à ses collègues en 1497 et 1498 au sujet du renouvellement des alliances avec Berne et Fribourg ${ }^{77}$, qui mentionnent précautionneusement qu'ils devaient s'y prendre "le plus doucement qu'ils pourront» ${ }^{78}$ et qu'ils devaient répondre "le plus doucement, amyablement et en la meilliour façon qu'ils pourront ${ }^{79}$.

Enfin, par ses ambassades, Aymon favorisa sa famille, en emmenant avec lui lors de certaines missions son neveu Pierre, qui fit partie de la légation qui alla chercher Marguerite d'Autriche en $1501^{80}$, tandis que son frère Georges l'accompagna à plusieurs reprises à Berne en 1497 et $1498^{81}$.

76. E. Pibiri, En voyage pour Monseigneur, p. 531-534.

77. ACV, CII 225 et CII 225, annexe.

78. ACV, CII 225, annexe, fol. 5.

79. ACV, CII 225, annexe, fol. 7.

8o. J. Dumont, Corps universel diplomatique du droit des gens, t. IV, partie I, p. 15; M. Bruchet, Marguerite d'Autriche, duchesse de Savoie, p. 31.

8r. AST, TGS, n 151, 1497-1498, fol. 114v-125r; AST, TGS, n 152, 1499-1500, fol. 167r-168r; F. Gabotto, Lo Stato sabaudo da Amedeo VIII ad Emanuele Filiberto, III, p. 84 sq., 106. 
Les liens privilégiés d'Aymon avec la ville confédérée ne se limitèrent d'ailleurs pas à la sphère diplomatique ${ }^{82}$, mais se concrétisèrent au niveau familial, peu après son décès, par un mariage entre sa nièce Jeanne qui épousa, en 1519, Christoph von Diesbach, fils de l'avoyer Wilhelm von Diesbach ${ }^{83}$, l'homme le plus fortuné de Berne, ambassadeur, tout comme Aymon, avec qui ce dernier avait traité en de nombreuses occasions. Une réussite, s'il en est.

Eva Pibiri

Université de Lausanne

82. En décembre 1494, l'avoyer de Berne, Rudolph von Erlach, sollicita Aymon de Montfalcon pour qu'il prenne son neveu à son service, signe des bonnes relations qui régnaient entre eux. Aymon accepta "pour l'amour dudict Seigneur avoyer son oncle» (P. Rück, «Un récit de la captivité du Chapitre de Lausanne en février 1537 », p. 60 sq.).

83. B. Braun-Bucher, "Diesbach, Wilhelm von». Wilhelm von Diesbach fut un fervent partisan de la politique francophile de Berne. Ses missions diplomatiques le conduisirent fréquemment en France et en Savoie; H. R., Schmidt, Worber Geschichte, p. 218. 


\section{BIBLIOGRAPHIE}

Andretta, Stefano, Péquignot, Stéphane, Waquet, Jean-Claude (éds), De l'ambassadeur. Les écrits relatifs à l'ambassadeur et à l'art de négocier du Moyen Âge au début du XIXe siècle, Rome, École française de Rome, 2015 (Collection de l'École française de Rome, 504).

Autrand, Françoise, "L'enfance de l'art diplomatique: la rédaction des documents diplomatiques en France, $\mathrm{XIV}^{\mathrm{e}}-\mathrm{XV}^{\mathrm{e}}$ siècles", in L'invention de la diplomatie, Moyen Âge-Temps modernes, éd. par Lucien Bély, avec la coll. d'Isabelle Rochefort, Paris, Presses universitaires de France, 1998, p. 207-224.

Autrand, Françoise, Contamine, Philippe, "Naissance de la France: naissance de sa diplomatie. Le Moyen Âge", in Histoire de la diplomatie française, présentation de Dominique de Villepin, Paris, Perrin, 2005, p. 41-156.

Bazzoli, Maurizio, "Ragion di stato e interessi degli stati. La trattatistica sull'ambasciatore dal XV al XVIII secolo", Nuova Rivista Storica, 86/2 (2002), p. 283-328.

Behrens, Betty, "Treatises on the Ambassadors written in the Fifteenth and Early Sixteenth Century", English Historical Review, 51 (1936), p. 616-627.

Braun-Bucher, Barbara, "Diesbach, Wilhelm von", in Dictionnaire historique de la Suisse, dir. par Mario Jorio, Hauterive, G. Attinger, 2005, vol. 4, p. 37.

Brero, Thalia, Rituels dynastiques et mises en scène du pouvoir. Le cérémonial princier à la cour de Savoie (1450-1550), Florence, Sismel-Edizioni del Galuzzo, 2017 (Micrologus Library, 84).

Bruchet, Max, Marguerite d'Autriche, duchesse de Savoie, Lille, Imprimerie L. Danel, 1927.

BüchI, Albert, Kardinal Matthäus Schiner als Staatsmann und Kirchenfürst. Ein Beitrag zur allgemeinen und schweizerischen Geschichte von der Wende des XV.-XVI. Jahrhunderts, I. Teil (bis 1514), Zürich, Kommissionsverlag Seldwyla, 1923. 
Charavay, Étienne, "Rapport sur les lettres de Louis XI et sur les documents concernant ce prince conservés dans les archives de l'Italie, adressé à M. le Ministre de l'instruction publique», Archives des missions scientifiques et littéraires, 7 (1881), p. 437-474.

Coutaz, Gilbert, "Le plan général de classement des Archives cantonales vaudoises de 1915: ses origines et ses développements. Deux siècles d'histoire institutionnelle", Rapport d'activité des Archives cantonales vaudoise, 2010, p. 37-141.

Crotti PAsi, Renata, "Filippo II, duca di Savoia", in Dizionario Biografico degli Italiani, 47 (1997), p. 766-770.

Dumont, Jean, Corps universel diplomatique du droit des gens..., Amsterdam, P. Brunel, R. et G. Wetstein/La Haye, P. Husson et Charles Levier, 1726, t. IV, partie I.

Gaвотто, Ferdinando, Lo Stato sabaudo da Amedeo VIII ad Emanuele Filiberto, Torino, 1893, t. II; Torino, 1895, t. III.

Helvetia Sacra, I/4. Le diocèse de Lausanne (VI siècle-1821), de Lausanne et Genève (1821-1925) et de Lausanne, Genève et Fribourg (depuis 1925), par un groupe d'auteurs, rédaction Patrick Braun, Bâle/ Francfort-sur-le-Main, Helbing \& Liechtenhahn, 1988.

Helvetia Sacra, I/5. Das Bistum Sitten/Le diocèse de Sion. L'archidiocèse de Tarentaise, rédaction Patrick Braun, Brigitte Degler-Spengler, Elsanne Gilomen-Schenkel, Basel, Schwabe Verlag, 2001.

Moeglin, Jean-Marie, "La place des messagers et des ambassadeurs dans la diplomatie princière à la fin du Moyen Âge», in Le diplomate en question ( $X V^{e}-X V I I I^{e}$ s.), éd. par Eva Pibiri, Guillaume Poisson, Études de Lettres, 286 (2010/3), p. 11-16.

Moeglin, Jean-Marie (dir.), PÉQuignot, Stéphane (coll.), Diplomatie et «relations internationales» au Moyen Âge (IXe-XVe siècle), Paris, Presses universitaire de France, 2017.

Molinet, Jean, Chroniques, éd. par Georges Doutrepont, Omer Jodogne, Bruxelles, Palais des Académies, 1935-1937, 2 vols.

Pibiri, Eva, «Histoire de femme, histoire d'État. Stratégie matrimoniale à la cour de Savoie pour la couronne de Chypre, 1455-1457", Bollettino storico-bibliografico subalpino, 102/2 (2004), p. 443472.

—, "Le personnel diplomatique d'Amédée VIII de Savoie entre France et Bourgogne au temps de la guerre de Cent Ans (1410-1440)", in La cour du prince. Cour de France, cours d'Europe (XII'-XVe siècle), 
éd. par Murielle Gaude-Ferragu, Bruno Laurioux, Jacques Paviot, Paris, H. Champion/Genève, Slatkine, 2011, p. 81-94.

—, En voyage pour Monseigneur. Ambassadeurs, offciers et messagers à la cour de Savoie (XIV $-X V^{e}$ siècles), Lausanne, Société d'histoire de la Suisse romande, 2011 (Mémoires et documents publiés par la Société d'histoire de la Suisse romande, $4^{\mathrm{e}}$ série/XI).

Queller, Donald E., Early Venetian Legislation on Ambassadors, Geneva, 1966 (Travaux d'Humanisme et Renaissance, 88).

Reymond, Maxime, "Aymon de Montfalcon, évêque de Lausanne, 1491-1517", Revue d'histoire ecclésiastique suisse, 14 (1920), p. 28-39 et 99-111.

Rотт, Édouard, Histoire de la représentation diplomatique de la France auprès des Cantons Suisses, de leurs alliés et de leurs confédérés, vol. I, 1430-1559, Berne, Imprimerie A. Benteli \& Co./Paris, Félix Alcan éditeur, 1900.

Rück, Peter, "Un récit de la captivité du Chapitre de Lausanne en février 1537 ", Revue historique vaudoise, 78 (1970), p. 43-67.

Santschi, Catherine, "Les annales du notaire montheysan Claude Revilliodi, 1490-1525», Vallesia, 23 (1968), p. 31-68.

Schmidt, Heinrich Richard, Worber Geschichte, Bern, Stämpfli Verlag, 2005.

Sсотт, Tom, The Swiss and their Neighbours, 1460-1560. Between Accommodation and Aggression, Oxford, Oxford University Press, 2017.

Segesser, Anton Philipp (Bearb.), Die Eidgenössischen Abschiede aus dem Zeitraum von 1500 bis 1520, Lucern, Meyersche, 1869 (Amtlichen Sammlung der Ältern Eidgenössischen Abschiede, Bd. 3/2).

Senatore, Francesco, "Uno mundo de carta": forme e strutture della diplomazia sforzesca, Napoli, Liguori, 1998 (Domini; Mezzogiorno medievale e moderno, 2).

Spitzbarth, Anne-Brigitte, Ambassades et ambassadeurs de Philippe le Bon, troisième duc Valois de Bourgogne (1419-1467), Turnhout, Brepols, 2013 (Burgundica, XXI).

Tremp, Ernst, "Arsent, François", in Dictionnaire historique de la Suisse, réd. en chef Mario Jorio, Hauterive, G. Attinger, 2002, vol. 1, p. 501. 
